\title{
Linking of Cell Tracks Using Segmentation
}

\author{
Mohammad Javeed, C Fathima Jabeen
}

\begin{abstract}
In maximum of image processing algorithms Segmentation is a key technique. It splits a digital image into several regions so as to examine them. Once segmentation is done, linking of frames is additionally is very important task. Several image segmentation techniques are developed by the researchers so as to form pictures swish and simple to judge. It is troublesome to process parallel algorithms in serial processors. This paper presents a literature review of basic image segmentation techniques, linking algorithms and want to process in hardware tools.
\end{abstract}

Key Words: Segmentation, Matlab, Verilog, linking, computer vision.

\section{INTRODUCTION}

The process of medical treatment is a growing and dynamic field with applications that expand into our daily life as medicine, explore areas, monitor, authenticate and many areas. Applications like these include quite a number of processes such as image enhancement and object detection. Application implementation on the basic configured PC is often more comfortable, but not very timeefficient because of additional memory limitations and different peripherals.

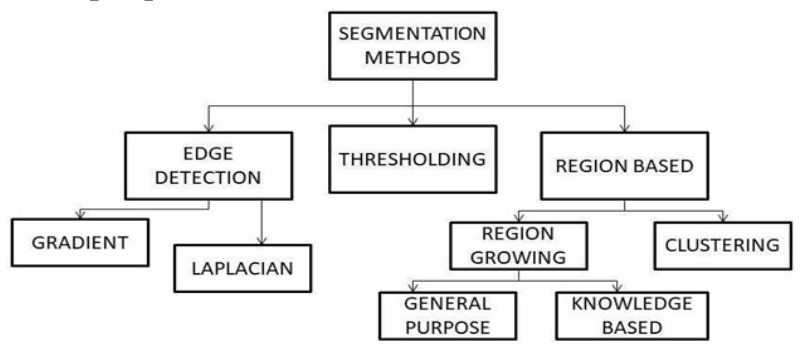

Figure 1 Segmentation methods

Application specific hardware implementation offers a sufficiently significant speed than the implementation of the softness system. While the VLSI technology for hardware has become a beautiful different system, it performs advanced computing tasks on hardware and implementation of parallelism and gas and gas algorithms that make the maximum drop at the time of execution.

There are two types of available technology for hardware design. A common design of a hardware design that is labeled as a specific integrated circuit (ASIC), which is programmed devices such as digital signal processors (DSPs) and field programming gates (FPGA). Full custom ASIC configuration offers most noteworthy execution, all things considered the multifaceted nature and moreover the value identified with the improvement is to a high degree high. The ASIC configuration can't be changed and furthermore the outline time is also terribly high. ASIC outlines are utilized in high volume modern applications.

Mohammad Javeed, Research Scholar, Dept. of ECE, Mewar University. Rajasthan, India.

Dr. Fathima Jabeen, Professor, Research Supervisor, Dept. of ECE, Mewar University, Rajasthan, India.
Revised Manuscript Received on October 15, 2019.

All through the outline creation the event of even one blunder makes the chip futile. DSPs are a kind of equipment gadgets that exists wherever between an ASIC and a PC regarding the execution and furthermore the plan perfection. DSPs are committed microchips, by and large customized in $\mathrm{C}$, or with gathering code for enhanced performance. It is comparative demeanor to extremely convoluted logical teach escalated errands like picture handling. Data of equipment configuration stays required, however the expectation to absorb information is route not as much as elective outline determinations.

Equipment plan systems like parallelism and pipelining strategies are frequently innovatively progressed on a FPGA, that isn't potential in devoted DSP outlines. Executing picture preparing calculations on reconfigurable equipment lessens an opportunity toadvertise esteem, permits quick prototyping of convoluted calculations and make more straightforward troubleshooting and confirmation. In this manner, FPGAs are a substitute for execution of continuous picture preparing frameworks.

The survey of division calculations are plainly said in [1], that are talking about inside the further segments. The following and connecting of cell tracks has unmistakably said in [2], that depends on Viterbi algorithmic program. The Viterbi algorithmic program is in [3].

\section{BASIC ALGORITHMS}

Algorithms used in automated cell tracking are commonly categorized as one of four categories: sequential tracking approach, particle filtering approaches, model-based approach evolution, Segmentation - An approach based on association and detection-based association approaches [4], [5], [6], [7], [8].

A few protest following at once should be possible by the Particle sifting technique. In numerous models like estimation models and dynamic models must be known before approximating the back appropriations of a question's current positions. Demonstrate based advancement approach initially creates the protest be followed and logically educates the deviations in its appearance. Dynamic shape strategies are not quite the same as the molecule separating techniques. This dynamic form strategy works fine notwithstanding for different molded articles. Identification based affiliation approach contain force thresholding, watershed calculations, morphological activities and angle (edge) discovery. However, because of power dissimilarities, picture commotion and antiques from the optical framework, force thresholding are not agent. In this strategy the thing is to portion and find the cells and next connection those items. Edge recognition shows enhanced 
execution than thresholding. However, on little differentiation pictures edge discovery is botch arranged to. The downside of watershed calculation is that it is arranged to commotion and much of the time over fragments the picture. As Smal et al. [9] and Li et al. [10] talked about molecule sifting with spatiotemporal data depends on the successive following methodology. Zhou et al.[11] talked about in their exploration on following of sperm cells which depends on an introduction versatile mean move advancement into molecule channel system. Beam et al. [12] connected with consecutive Bayesian structure to set up cell correspondences. Ryoo and Aggarwal et al. [13] proposed a computationally efficient calculation for following lower than unadorned impediment. Different consecutive following techniques clarified above are computationally serious and later experience the ill effects of the trouble of adaptability.

Segmentation-based outline by-outline affiliation approach is additionally agent for mechanized cell following. Al-Kofahi et al. [14] utilize versatile thresholding technique, Liu et al. [15] offered the Hidden Conditional Random Field Model. In any case, these methodologies don't show the season of consummation of cell division and they are computationally escalated. Dynamic Contour display [10], Watershed strategy [16], Adopting Motion Model [10] has been generally utilized for cell following [17].

\section{COMPUTER VISION BASED ALGORITHMS}

We will talk that by counting the following methods of stack in this area, it has been adjusted to build a computerized, stack-set framework, focusing on the application. A following structure in casing to outline in light of track assemblage and track connecting and interface the track directions so found to build up the whole heredity tree talked about by Kanade et al. [18]. Numerous Model Filters (IMM) in following natural occasions the Communicating are additionally appeared in this strategy which are enhanced execution than Kalman Filter.

By developing a machine learning strategy which ascertains a vitality term in the level-set based cell tracker and enhances the following execution has talked about by $\mathrm{Li}$ et al. [10] have very much followed undeveloped cells. To decrease an edge based approach and clamor level Bilateral sifting was utilized adequately in cell finder is dynamic to catch divergent stages in mitosis. In division chart cut technique from Yang et al. [19] proposed. The have talked about on following cerebrum cells, as this strategy confirmations to get all around best arrangement. Huh et al. [20] tells that following based strategy, following free technique are two writes in robotized mitosis discovery calculations. Following by closest neighbor approach, Park et al. [21] examined on single mRNA following in live cells and received molecule computerized following of mRNA in cells Multiple Hypothesis Tracking (MHT) calculations and Kalman Filtering. Kanade et al. [4] sought after their work further and introduced a picture reclamation and division strategy which utilizes a quadratic streamlining capacity to reestablish the first picture dispensing with the relics like shadows, shading and radiances. Thresholding gives excellent execution while dividing the reestablished picture.
Moreover, a three stage approach is additionally exhibited for identification of cell division (mitosis), which incorporates making patch arrangement, removing highlights lastly distinguishing whether the fix contains a mitosis event and the particular area of occurrence.

Scherf et al. [22] displayed a calculation to naturally figure attributes of single cells in a profoundly thick populace and develop its whole genealogy history. This calculation likewise utilized levelset division for question recognition and watershed calculation was connected to ease the issue of cell impediment. Padfield et al. [8] presented the chart theoretic least cost stream structure to determine the information affiliation which was precise in cell following. Notwithstanding, this technique fizzled for covering cells As an answer for this issue Bise et al. [7] proposed the shape following strategy in light of halfway form coordinating which could recognize various covering cells. This procedure utilizes form states of cells and bunches and expertly fuses it to track covering cells of Human Central Nervous System foundational microorganisms amid relocation and expansion [7].

Kang et al. [23] proposed a novel technique for following cores of C.elegans amid embryogenesis. The calculation comprises of two viewpoints: following of cores by utilizing a basic circular veil, and identification of cell division by following different questions locally and recognizes the new conceived sister cells at a later purpose of time when its reality turns out to be all the more clear with a specific end goal to stay away from false discovery. This calculation demonstrates exact and hearty following outcome even in low Signal-to-Noise Ratio (SNR) pictures. Yuan et al. [24] have said that Mean-move calculation is a quick and effective protest following technique and has been effectively connected to observation and division framework. To dispense with the issue of consolidating, part protests and vanishing Jaqaman et al. [25] expected a calculation, the direct task issue (LAP). In view of the Kalman channel Yang et al. [26] anticipated a calculation for predictable following of vast scale thick antiparallel molecule flag. These calculations are not appropriate for low Signal-to- Noise Ratio. In this manner, Smal et al. [9] introduced a capable calculation utilizing molecule sifting which was equipped for following articles in uproarious picture arrangements. However, this system utilizes non specific molecule separating (GPF), which is executable costly. Sargin et al. [27], Koulgi et al. [28] characterized divergent strategies to track the shortening, lengthening, coasting shrouded Markov demonstrate and a graphical model-based calculation, individually. The constraint of this approach was that it just functions admirably for little relocations. The most recent improvement in the field of question following is the utilization of kymographs. Kymograph connotes the movement data in a solitary diagram which is an essential technique. However, in this once the protest moves rapidly, irregular courses are made and the technique neglects to give the coveted show. Yuan et al. [24], in his work on following axons, he utilized

Published By:

Blue Eyes Intelligence Engineering 
molecule separating approach. The possibility of him was to energetically bound the spatial space to the state of the axon. The way of the axon movement is created with a piecewise polynomial capacity communicated utilizing cubic spline interjection. Huang et al. [29] built up a 3-D online framework for checking and following of cells in a suspension.

Without express target identification in a succession of picture a novel technique for visual following of different targets exhibited by Hoseinnezhad et al. This calculation depends on multi-Bernoulli channel. This strategy extends most exceptional outcomes when down to earth to numerous objectives with parallel visual example. In like manner, it represents targets coming in and leaving the scene. In minuscule pictures a calculation for different targets following for following cells have proposed by Magnusson et al. [30]. Viterbi calculation is utilized as a part of this technique which was connected to the picture grouping and worldwide information affiliation is achieved due to here information from the entire picture is used to settle on nearby choices about working of the cell tracks.

To assess the calculation tests were performed on 115 picture groupings of Muscle Stem Cells (MuSCs) and the accuracy and review rate (called mitosis expanding rightness) registered by the calculation was seen to be to 0.78 and 0.75 for mitosis (cell division occasion) 0.74 and 0.62 for apoptosis (cell passing occasion), when contrasted with Huh et al. [20] the review rate was 0.65. In light of a worldly probabilistic model a mechanized mitosis location calculation proposed by Huh et al. [31] for approval of mitosis occasion recognition. The forecast was accomplished god comes about as $97.4 \%$ exactness and 96.6\% continued 14 cell populaces of Hematopoietic Stem cells (HSC) and it was watched that $97.4 \%$ accuracy. Chatterjee et al. [6] proposed a robotized arrangement of following human monocyte cells in a video succession in which first the picture is preprocessed to dispense with any foundation clamor and the situation of centroids of the cells is acquired. At that point the Maximum Cardinality Minimum Weight Bipartite Matching is connected to make the cell direction. Mkrtchyan et al. [32] built up an effective procedure for utterly computerized enrollment of picture and compared it to images of shoot apical meristem (SAM) of Arabidopsis to assess its execution and watched that this calculation enhances cell heredity and information measurements. The way toward changing distinctive arrangements of information is Image enlistment into one organize framework.

From these distinctive estimations so as to have the capacity to look at or coordinate the information got enlistment is required. They received Iterative Closest Point calculation with slight change. Highlights of the nearby neighborhood zone to stamp the relating milestone sets utilized as a part of this case IPC utilizes for picture enrollment. Cell following and recognizes the right cell way taking out impediment occasions precisely proposed by Baker et al. [33]. This calculation consolidates four modules: connection examination, cell following, impediment recognition and revision and cell and kin distinguishing proof. Here shape based division is utilized. Konda et al. [34] attempted to report the trial of following a

populace of cells which display varieties in conduct, flow and thickness. They anticipated an Event Indicator Function classifier (ElF) for recognizing cell following flaws and phenotypes. The EIF is intended to explicitly perform to play out the numerous picture handling procedures. Hagwood et al. [35] assessed execution of four general division calculations: Otsu ,K-implies, Canny, and Watershed in view of their misclassification blunder. Investigations were done as grouping of pictures of A10 rodent smooth muscle cells and NIH-3T3 cells and chose that Canny and Watershed were solid and genuine for an inconstancy of tests.

\section{AUTOMATIC CELL TRACKING \& RESULTS}

Kanade et al. [18] acquainted An Automated Cell Tracking System for simultaneously following a great many cells utilizing stage differentiate time-pass microscopy . In this technique five modules were incorporated: dynamic channel, cell tracker, cell finder, track linker and track compiler to achieve spatiotemporal connecting of cell ways. The framework execution was evaluated and accomplished precision in the scope of $85.9 \%$ and $92.5 \%$, which is $9 \%$ more rather than the former framework. Li et al. [10] built up a PC vision based cell following framework that is equipped for following the execution of an extensive populace of cells and in addition cell development, demise , tranquility, division and separation. In this strategy the examinations he beat the numerous perspectives like segregating between covering cells, expanding cell densities amid populace development and cell entering/leaving the field of.

A framework for cell following is outlined which depends on shape based cell following in Bise et al. [17]. The structure was utilized for exhaustive investigation of Human Nervous System undifferentiated cells and accomplished 97\% precision. In this framework each picture succession experiences three stages: cell-blob correspondence preconditioning and division and partition of covering cells. Yang et al. [19] examined Tracking and Segmentation Framework to defeat limit vulnerability issue and utilized it to digest 2D shape limits from Serial Block Face Scanning Electron Microscopy picture stacks. In this strategy chart slice framework was used to get the overall best arrangement of a vitality work which includes the motion of the inclination vector handle, the separation work and the picture dim scale force. This vitality capacity can take care of the limit vagueness issue happening in thickly pressed EM pictures. A Real-time Cell Tracking System was mechanically best in class which could gauge cell relocation courses underneath cell culture circumstances. The results are single cell way $(\mathrm{x}, \mathrm{y})$ amid relocation, movement speed, relocation remove, cell estimate, continuous pictures and bounty. This framework is suitable to a wide range of looks into identified with cell relocation, for example, cell angiogenesis, chemo taxis, and besides disease metastasis [36]. A robotized framework capable of including cell multiplication measurements vitro progressively was created 
by Kandade et al. [4] which was fortified online regular examinations. Three cell picture examination calculations: picture rebuilding, mitosis recognition and blunder tolerant information affiliation was started into an open site. Analysts the whole way across the world can transfer their cell pictures for preparing. The framework's execution was tried to be exceptionally productive. Magnusson et al. [30] depicts the above structure as seemingly the most developed framework right now. Bise et al. [7] proposed a mechanized strategy to acquire cell directions and genealogies which was turned out to be more productive than the past technique that utilized level- set method. Test comes about on a testing informational collection demonstrate that the proposed technology altogether enhances the following execution including target adequacy, track virtue; mitosis stretching accuracy by comprehensively partner tracklets.

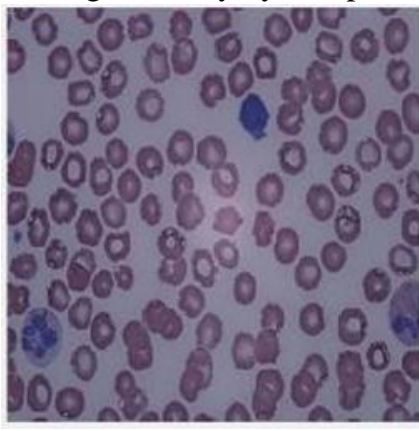

a.

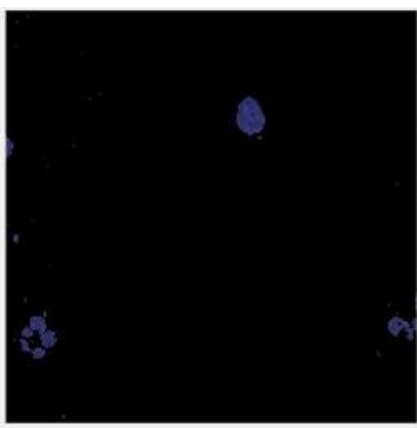

b.

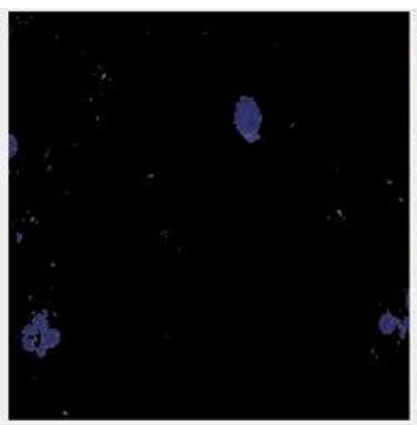

c.

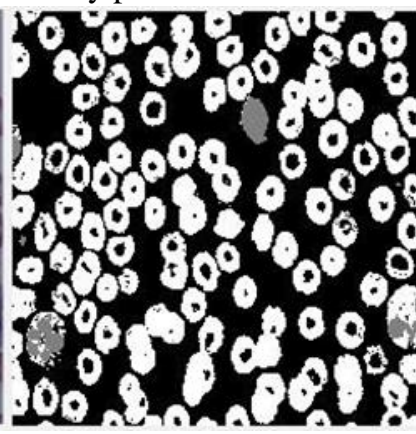

d.

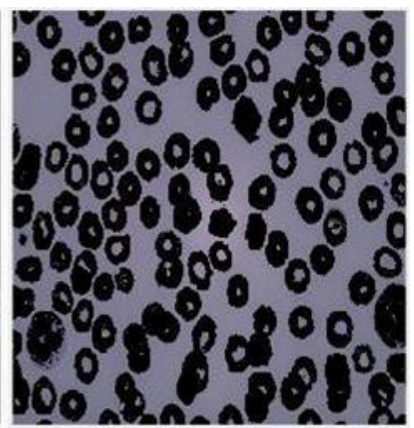

e.

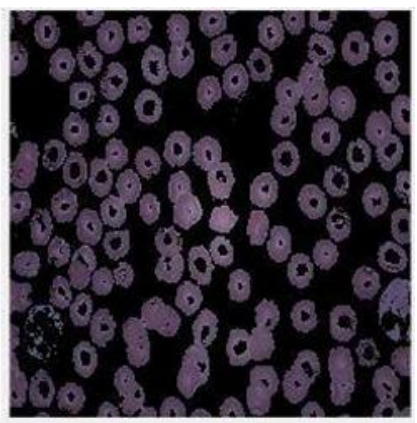

f.
Figure 2 example of segmentation of a cell a. original image, b. Segmented image, d. Intermediate image, (c)(e)(f) objects in clusters.

The utilization of convolutional codes with probabilistic deciphering might permanently move forward the lapse execution of a correspondence framework[37]. Trellis coded modulation schemes are utilized within numerous data transfer capacity proficient frameworks. Regularly a TCM framework uses a helter skelter rate convolutional code, which prompts secondary intricacy from claiming viterbi decoder for those TCM decoder, At those demand length for Convolutional code is Additionally typical. For case the rate $3 / 4$ convolutional code utilized within trellis coded regulation framework for whatever requisition need a demand period from claiming 7 will be in the unpredictability of the comparing viterbi decoder for An rate $1 / 2$ convolutional code with demand period from claiming 9 [38] because of those vast number of moves in the trellis. So, As far as force consumption, those viterbi decoder may be overwhelming module On a TCM decoder.

Set up should diminishing the individuals computational many-sided nature What is more also control consumption, low drive schemes ought should make abused of the vd Previously, an TCM decoder. All results to flat energy viterbi decoder outline will be mulled over to our usage fill in. Force diminishment over VDs Might make attained by decreasing the number about states, (for instance diminished state succession deciphering [37], M-algorithm [39] and Talgorithm [37],[40],) alternately by again scaling those supply voltage[41]. Again mounting of the supply voltage will be Hosting an issue that it needs with take entire framework under thought including with vd at which we would not centering from claiming our Look into. For useful provision RSSD will be additional regularly utilized over M-Algorithm which will be by and large not Likewise useful Likewise M-algorithm [39] and T-Algorithm. Essentially M- Algorithm obliges An foray methodology for a sentiment circle while $\mathrm{T}$ - calculation main searches to the ideal way metric that is those most extreme quality alternately the base esteem of Ps.

T-Algorithm Need been demonstrated will precise productive in decreasing the control utilization [42], [43]. However, seeking for those ideal way metric in the sentiment circle still lessens those deciphering velocity. Will beat this drawback, T- Algorithm need suggested over two variations, the loose versatile vd [44], Which infers utilizing a evaluated ideal way metric, As opposed to finding those genuine person every cycle and the limited-search parallel state vd In view of rare state move [SST][45].

At connected will secondary rate convolutional codes, the loose versatile vd suffers An extreme debasement of BitError-Rate(BER) execution because of those intrinsic floating slip the middle of the evaluated ideal way metric and the exact one[46]. On the great holders kept all those SST based plan obliges pre deciphering Furthermore re encoding transform What's more may be not suitableness for TCM decoders. For TCM, the encoded information would continuously connected with an intricate multilevel regulation plan such as 8 -ary stage shift keying (8PSK) or 16/64-ary quadrature abundancy tweak (16/64QAM) through An heavenly body purpose mapper. At the receiver, a delicate enter vd if a chance to be utilized with assurance a great coding addition. So, those computational through leader and deciphering inactivity because of pre deciphering What's more re encoding of the TCM indicator turned into secondary. An add-compare select unit (ACSU) building

Published By:

Blue Eyes Intelligence Engineering \& Sciences Publication

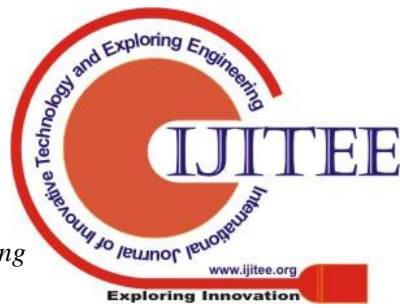


design In view of pre calculation for VDs incorporating TAlgorithm [46], which proficiently enhances the clock pace of a vd for T-Algorithm for An rate $3 / 4$ code. Now, we further dissect those pre calculation calculation. A precise path with determine those ideal pre calculation steps will be shown, the place the least amount of steps for incredulous way on accomplish the hypothetical cycle bound may be ascertained and the computational multifaceted nature overhead because of pre calculation will be assessed. Later, The complete low-power VD design for the rate $3 / 4$ convolutional code discussed in [37], [47]

\section{CONCLUSION}

In this we have clearly discussed the segmentation methods and the advantages and disadvantages of the different techniques. Segmentation and linking based on the Viterbi algorithm is taking interest because linking after segmentation is also having the most importance in medical image processing to segment the blood cells. Viterbi algorithm for convolution codes decoding having a vital role in the encoding and decoding processes. Linking of the cells with Viterbi algorithm after segmentation is having more advantages as it has decreased the linking time and power when we execute this algorithm in hardware tools.

\section{REFERENCES}

[1]. Akhilesh Arora, Tayeba Qazi" Computer Vision Based Tracking Of Biological Cells-A Review" International Conference of Advance Research and Innovation (ICARI-2014). ISBN 978-935156-328-0.

[2]. Klas E. G. Magnusson*, Student Member, IEEE, Joakim Jaldén, Senior Member, IEEE, Penney M. Gilbert, and Helen M. Blau, "Global Linking of Cell Tracks Using the Viterbi Algorithm”, IEEE TRANSACTIONS ON MEDICAL IMAGING, VOL. 34, NO. 4, APRIL 2015

[3]. K. S. Arunlall and Dr. S. A. Hariprasad2" An Efficient Viterbi Decoder", International Journal of Advanced Information Technology (IJAIT) Vol. 2, No.1, February 2012.

[4]. T. Kanade, Z. Yin, R. Bise, S. Huh, S. Eom, M. Sandbothe, F. M. Chen, "Cell Image Analysis: Algorithms, System and Applications," pp. 374-381, 2010

[5]. K. L. K. Li, E. D. Miller, L. E. Weiss, P. G. Campbell, T. Kanade, "Online Tracking of Migrating and Proliferating Cells Imaged with Phase-Contrast Microscopy," 2006 Conf. Comput. Vis. Pattern Recognit, Work. pp. 0-7, 2006

[6]. R. Chatterjee, M. Ghosh, A. S. Chowdhury, N. Ray, "Cell tracking in microscopic video using matching and linking of bipartite graphs" Comput. Methods Programs Biomed, 112(3), pp. 422-31, Dec. 2013

[7]. R. Bise, Yin. Zhaozheng, T. Kanade, "Reliable cell tracking by global data association," Biomedical Imaging: From
Nano to Macro, 2011 IEEE International Symposium on, pp.1004,1010, March 30 2011-April 2011

[8]. Padfield, , J. Rittscher, B. Roysam, "Coupled minimumcost flow cell tracking for highthroughput quantitative analysis.," Med. Image Anal., 15(4), pp. 650-68, Aug. 2011

[9]. Smal, K. Draegestein, N. Galjart, W. Niessen, E. Meijering,Particle filtering for multiple object tracking in dynamicfluorescence microscopy images: application to microtubulegrowth analysis, IEEE Transactions on Medical Imaging 27 (6)(2008) 789-804.

[10]. K. L. K. Li, E. D. Miller, M. C. M. Chen, T. Kanade, L. E. Weiss, P. G. Campbell, "Computer vision tracking of stemness," 2008 5th IEEE Int. Symp. Biomed. Imaging from Nano to Macro, pp. 0-3, 2008

[11]. X. Zhou, Y. Lu, Efficient mean shift particle filter for spermcells tracking, International Conference on ComputationalIntelligence and Security (2009) 335-339

[12]. N. Ray, G. Dong, S.T. Acton, Tracking multiple cells bycorrespondence resolution in a sequential bayesianframework, Proceedings of IEEE Conference on ImageProcessing (2005) 705708.

[13]. D. Chetverikov, J. Veresto'y, "Feature point tracking forincomplete trajectories, computing", Devoted Issue on Digital Image Processing 62 (1999) 321-338

[14]. O. Al-Kofahi, R. Radke, S. Goderie, Q. Shen, S. Temple, B. Roysam, "Automated cell lineage construction: A rapid method to analyze clonal development established with murine neural progenitor cells," Cell Cycle, 5(3), pp. 327-335, 2006

[15]. Liu et al., "Mitosis Sequence Detection using Hidden Conditional Random Fields," IEEE International Symposium on Biomedical Imaging (ISBI), 2010

[16]. L. Vincent, P. Soille, "Watersheds in digital spaces: an efficient algorithm based on immersion simulations", IEEE Trans. Pattern Anal. (1991) 13(6) 583-598

[17]. R. Bise, K. Li, S. Eom, T. Kanade, "Reliably Tracking Partially Overlapping Neural Stem Cells in DIC Microscopy Image Sequences," pp. 67-77, 2009.

[18]. K. L. K. Li, T. Kanade, "Cell Population Tracking and Lineage Construction Using Multiple-Model Dynamics Filters and Spatiotemporal Optimization,” Proc. 2nd Work. Microsopic Image Anal with Appl. Biol. Piscataway, NJ, USA, pp. 1-8, 2007.

[19]. Yang, Huei-Fang, Y. Choe, "Cell tracking and segmentation in electron microscopy images using graph cuts," Biomedical Imaging: From Nano to Macro, 2009, ISBI '09. IEEE International Symposium on, pp.306, 309, June 28 2009-July 1 2009. 


\section{Linking of Cell Tracks Using Segmentation}

[20]. S. Huh, S. Eom, , R. Bise, Z. Yin, T. Kanade, "Mitosis Detection for Stem Cell Tracking in Phase-contrast microscopy images," Robotics Institute, Carnegie Mellon University," pp. 2121-2127, 2011

[21]. Y. Park, A. R. Buxbaum, R. H. Singer, "Single mRNA Tracking in Live Cells", 1st ed., vol. 472, no. 10. Elsevier Inc., 2010, pp. 387-406

[22]. N. Scherf, K. Franke, I. Glauche, I. Kurth, M. Bornh, "On the symmetry of siblings : automated single-cell tracking to quantify the behavior of hematopoietic stem cells in a biomimetic setup," pp. 119-130, 2012

[23]. S. Kang, C. A. Giurumescu, A. D. Chisholm, P. Cosman, "Automated nuclei tracking in C. elegans based on spherical model fitting with multiple target tracking," Image Analysis and Interpretation (SSIAI), 2012 IEEE Southwest Symposium on, pp.17,20, 22-24 April 2012

[24]. L. Yuan, S. Member, Y. F. Zheng, J. Zhu, L. Wang, A. Brown, "Object Tracking With Particle Filtering in Fluorescence Microscopy Images: Application to the Motion of Neuro fi laments in Axons," 31(1), pp. 117-130, 2012

[25]. K. Jaqaman, D. Loerke, M. Mettlen, H. Kuwata, S. Grinstein, S.Schmid, and G. Danuser, "Robust single-particle tracking in live-cell time-lapse sequences," Nature Methods, 5(8), pp. 1212- 1221, 2008.

[26]. G. Yang, A. Matov, G. Danuser, "Reliable tracking of large scale dense antiparallel particle motion for fluorescence live cell imaging," in Proc. IEEE Conf. Comput. Vis. Pattern Recognit., San Diego, CA, 2005, pp. 9-17

[27]. M. E. Sargin, A. Altinok, K. Rose, B. S. Manjunath, "Deformable trellis: Open contour tracking in bio-image sequence," in Proc. IEEE Int. Conf. Acoustics, Speech, Signal Process., Las Vegas, NV, 2008, pp. 561-564

[28]. P. Kougli, M. E. Sargin, K. Rose, B. S. Manjunath, "Graphical model-based tracking of curvilinear structures in bioimage sequences," in Proc. IEEE Int. Conf. Pattern Recognit., Istanbuk, Turkey, 2010, pp. 2596-2599

[29]. Huang, S. Sankaran, D. Racoceanu, S. Hariharan, S. Ahmed, "Online 3-D Tracking of Suspension Living Cells Imaged with Phase-Contrast Microscopy,” 59(7), pp. 1924-1933, 2012

[30]. G. Magnusson, E. Klas, J. Jald, “A Batch Algorithm Using Iterative Application of the Viterbi Algorithm to track cells and construct cell lineages" access Linnaeus Center, Signal Processing Lab , KTH Royal Institute of Technology, Stockholm , Sweden," pp. 382-385, 2012

[31]. S. Huh, S. Eom, Fei, E. Ker, L. Weiss, T. Kanade, "Mitosis Detection of Hematopoietic Stem cell populations in time-lapse phasecontrast microscopy images Robotics Institute , 2
Department of Biological Sciences and Biomedical Engineering, Carnegie Mellon University,” pp. 390-393, 2012.

[32]. K. Mkrtchyan, A. Chakraborty A. K. R. Chowdhury, "Automated registration of live imaging stacks of Arabidopsis," Biomedical Imaging (ISBI), 2013 IEEE 10th International Symposium on, pp.672, 675, 7-11 April 2013

[33]. R. M. Baker, M. E. Brasch, J. H. Henderson, M. L. Manning, "Contour-Based Algorithm for Tracking Cells and CellMaterial Analyses," 2013 39th Annu. Northeast Bioeng. Conf., pp. 96-97, Apr. 2013

[34]. R. Konda, R. Chakravorthy, S. Challa, "Design and analysis of an Event Indicator Function classifier for immune cell tracking applications,” 2013 IEEE/ASME Int. Conf. Adv, Intell Mechatronics, pp. 199-205, Jul. 2013

[35]. C. Hagwood, J. Bernal, M. Halter, J. Elliott, "Evaluation of Segmentation Algorithms on Cell Populations Using CDF Curves," Medical Imaging, IEEE Transactions on, 31(2), pp.380, 390, Feb. 2012

[36]. Y. H. Kwak, S. M. Hong, S. S. Park, "A single cell tracking system in real-time.," Cell. Immunol, 265(1), pp. 44-9, Jan. 2010.

[37]. F. Chan and D. Haccoun, "Adaptive viterbi decoding of convolutional codes over memory less channels" IEEE Trans. Commun. , vol. no. 45, 11, pp. 1389-1400, Nov. 1997. "Bandwidth- efficient modulations" Consultative Committee For Space Data System, Matera, Italy, CCSDS 401(3.3.6) Green Book, Issue 1, Apr. 2003.

[38]. J. B. Anderson and E. Offer, "Reduced-state sequence detection with convolutional codes" IEEE Trans. Inf. Theory, vol. 40, no. 3, pp. 965-972, May 1994.

[39]. C. F. Lin and J. B. Anderson, “ T-algorithm decoding of channel convolutional codes," presented at the Princeton Conf. Info. Sci. Syst., Princeton, NJ, Mar. 1986.

[40]. S. J. Simmons, "Breadth-first trellis decoding with adaptive effort”IEEE Trans. Commun. , vol. 38, no. 1, pp. 3-12, Jan. 1990.

[41]. J. Jin and C.-Y. Tsui, "Low-power limited-search parallel state viterbi decoder implementation based on scarece state transition" IEEE Trans. Very Large Scale Integr. (VLSI) Syst., vol. 15, no. 11, pp. 1172-1176, Oct. 2007.

[42].

F. Sun and T. Zhang,

"Low power state-parallel 
relaxed adaptive viterbi decoder design and implementation," in Proc. IEEE ISCAS, M ay 2006, pp. 4811-4814.

[43]. J. He, H. Liu, and Z. Wang, "A fast ACSU architecture for viterbi de-coder using T-algorithm," in Proc. 43rd IEEE Asilomar Conf. Signals,Syst. Comput. , Nov. 2009, pp. 231-235.

[44]. Md.Javeed, B.Sri Lakshmi," Low power Viterbi decoder for Trellis coded Modulation using T-algorithm”, international journal of scientific \& engineering research, volume 3, issue 9, september-2012 .

[45]. First MD. JAVEED, Second B. HARI KRISHNA, Jr., and Third M.V.S.R KISHORE, "low power viterbi decoder design for tcm decoders using t-algorithm",CIIT international journal of programmable devices, November,2012.

[46]. Mohammad Javeed1 and Battula Swapna2, "High speed Convolution encoder and Viterbi decoder using dynamic shift register" International Journal of Electronic and Electrical Engineering”, ISSN 0974-2174, Volume 7, Number 5 (2014), pp. 483-490.

[47]. Abdul Manan, "Implementation of Image Processing Algorithm on FPGA", Department of Electronics and Communication Engineering, Ajay Kumar Garg Engineering College, PO Adhyatmic Nagar. 